\title{
Neurotrauma admissions and COVID-19: a National Centre experience
}

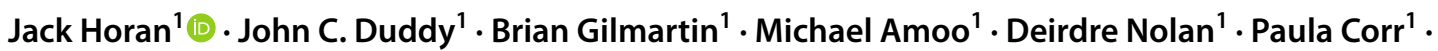 \\ Mohammed Ben Husien ${ }^{1}$. Ciaran Bolger ${ }^{1}$
}

Received: 14 August 2021 / Accepted: 11 September 2021 / Published online: 23 September 2021

(c) Royal Academy of Medicine in Ireland 2021

\begin{abstract}
Background To investigate the impact of COVID-19 on trauma admissions to a National Neurosurgical Centre in Ireland. Methods Retrospective analysis of a prospectively maintained database of all trauma admissions to the National Neurosurgical Centre at Beaumont Hospital, Dublin, during the period March 1 to May 31, 2019 and 2020. Primary outcome was 30-day mortality rate. Secondary outcomes included time transfer time, time from admission to time of surgery, and intensive care unit (ICU) admissions. Patients under the age of 16 were excluded.

Results A total of 32 and 39 patients were admitted to the National Neurosurgical Centre following trauma over the 3-month period in 2020 and 2019 respectively, giving a $17.9 \%$ reduction in admissions. The 30-day mortality rate increased from $7.7 \%$ in 2019 to $15.6 \%$ on 2020 ( $p=0.45$ ). Mean transfer time was $4 \mathrm{~h} 58 \mathrm{~min}$ in 2019 and $3 \mathrm{~h} 55 \mathrm{~min}$ in 2020 (0.22). Mean time from admission to time of surgery was $9 \mathrm{~h} 10 \mathrm{~min}$ in 2019 and $5 \mathrm{~h} 37 \mathrm{~min}$ in 2020 respectively $(p=0.35)$. In 2019, 20 patients (51.3\%) were admitted to ICU. This increased to 23 patients $(69.7 \%)$ in $2020(p=0.08)$.

Conclusions Traumatic brain injury 30-day mortality rates increased during the first COVID-19 lockdown period. Trauma admission rates to ICU remained unchanged despite an overall reduction in trauma admissions. Transfer time, time to surgery, and length of stay were impacted by COVID-19. Despite the challenges COVID-19 has posed, it is important to maintain a fully functioning neurosurgical and neurocritical care service during the pandemic.
\end{abstract}

Keywords COVID-19 $\cdot$ Neurotrauma $\cdot$ Mortality $\cdot$ Trauma $\cdot$ Traumatic brain injury

\section{Introduction}

Traumatic brain injury (TBI) and spinal injury have significant burdens on society, beyond disability and mortality rates. In high-income countries, TBI is the most significant contributor to death and disability in young people [1]. In Europe, it is estimated that there are 82,000 deaths per year due to TBI [2]. TBI requiring admission to an intensive care unit (ICU) has a considerable mortality of between 26 and $45 \%$ [3-5]. Worldwide, the aetiology of TBI and spinal injury has changed. Road traffic collisions (RTC) were the leading cause of trauma over 25 years ago, with low energy falls in the elderly from a standing height now the predominant mechanism of trauma in Ireland [6].

Jack Horan

jackhoran27@gmail.com

1 Department of Neurosurgery, Beaumont Hospital, Dublin 9, Ireland
COVID-19 has impacted every country worldwide since it was first reported in December 2019. The first confirmed Irish case of COVID-19 was in late February 2020 [7]. With cases increasing significantly, Ireland entered its first period of lockdown to try and suppress virus transmission on 12 March. These restrictions included school closures, working from home for all non-essential businesses, no social gatherings, and to stay within $2 \mathrm{~km}$ of the home [8]. Hospitals were required to reorganise many components including staffing, repurpose clinical areas to make-shift intensive care units (ICU), and the cancelling of outpatient appointments. These restrictions were relaxed on 18 May 2020, with some sectors returning to work and the gradual opening of schools and non-essential businesses [9]. Our group has previously shown that referrals to the national neurosurgical centre were reduced during the lockdown period of March-May 2020 [10]. Other studies showed that trauma and emergency presentations decreased by up to $50 \%$ across neurosurgery, other surgical specialities, and emergency departments as a result of lockdowns imposed by governments [11-13]. 
However, the impact of COVID-19 on the mortality of trauma admissions to neurosurgical and neuro-ICU units has not been well studied. We conducted a retrospective cohort study that investigated the impact COVID-19 has had on patient outcomes following trauma in those admitted to a National Neurosurgical Centre.

\section{Methods}

We performed a retrospective analysis of a prospectively maintained database of all trauma admissions to the National Neurosurgical Centre at Beaumont Hospital, Dublin, during the period March 1 to May 31, 2020, and compared it to the same period in 2019. Beaumont Hospital is the national neurosurgical centre and provides sub-speciality care to the entire Republic of Ireland, a population of approximately 4.8 million people [14].

The primary outcome of the study was to assess the impact COVID-19 had on mortality rates in trauma cases admitted to the National Neurosurgical Centre. Key performance indicators (KPIs) are used in our institution as part of ongoing quality improvement. We have included these as secondary outcomes, and they are time from acceptance of patient to admission (transfer time) and time from admission to time of surgery.

Patient characteristics including age, gender, alcohol use, anticoagulant/antiplatelet use, initial Glasgow Coma Scale (GCS), intubation and ventilation status at referral, surgical intervention, ICU admission, and length of stay (LOS) were recorded. Radiological diagnoses of TBI were recorded based on formal radiological reporting by a consultant radiologist. The trauma aetiology was included and classified as fall, high fall (fall from above $2 \mathrm{~m}$ and above), road traffic collision, bicycle injury, and alleged assault. Patients under the age of 16 were excluded as the paediatric ward was closed for part of the 2020 time period.

\section{Statistical analysis}

Statistical analysis was performed using SPSS version 24.0 (SPSS, Chicago, Illinois, USA) on the study group. Categorical variables were analysed using frequency tables, and comparisons were made using the chi-squared test or Fisher's exact test where appropriate. Quantitative variables were analysed using the independent $t$-test. All tests were two-tailed and for tests of significance; $p<0.05$ was considered statistically significant.

\section{Results}

A total of 32 and 39 patients were admitted to the National Neurosurgical Centre following trauma over the 3-month period in 2020 and 2019 respectively. Study outcomes including 30-day mortality, transfer time, admission to surgery time, LOS and patient characteristics, and trauma aetiology are shown in Tables 1-3.

\section{Diagnoses}

The trauma diagnoses are shown in Table 2. Many patients had more than one pathology, and so the total number of diagnoses is greater than the total number of patients in both years.

\section{GCS}

Median initial GCS was 13 in 2019 and 9 in 2020. Mean initial GCS was lower in 2020 than 2019 (9.1 vs 10.6) but did not reach statistical significance $(p=0.19)$. The proportion of patients with a severe TBI (GCS 3-8) in 2019 was $43.3 \%$ and $24.1 \%$ in 2020. Moderate TBI (GCS 9-13) accounted for $13.4 \%$ and $31.0 \%$ patients in 2019 and 2020 respectively. Mild TBI (GCS 14 and 15) were similar in proportions with 43.3 and $44.8 \%$ of patients in 2019 and 2020 being referred with a GCS of 14 or 15 .

Median GCS for mild TBI was 15 in 2019 and 2020. Moderate TBI median GCS was 13 in 2019 and 9 in 2020

Table 1 Patient demographics

\begin{tabular}{llll}
\hline & $\mathbf{2 0 1 9}$ & $\mathbf{2 0 2 0}$ & $\boldsymbol{p}$ value \\
& $n=39$ & $n=32$ & \\
\hline Gender & & & \\
Male & $25(64.1 \%)$ & $21(65.6 \%)$ & \\
Female & $14(35.9 \%)$ & $11(34.4 \%)$ & \\
Age (Years) mean & $54.6(18-82)$ & $45.8(19-78)$ & \\
Alcohol use & $4(10.3 \%)$ & $5(15.6 \%)$ & \\
Antiplatelet/anticoagulant & $5(12.8 \%)$ & $1(3.1 \%)$ & \\
GCS median (initial) & $13(3-15)$ & $9(3-15)$ & 0.19 \\
GCS 3-8 & $43.3 \%$ & $24.1 \%$ & \\
GCS 9-13 & $13.4 \%$ & $31.0 \%$ & \\
GCS 14 and 15 & $43.3 \%$ & $44.8 \%$ & \\
GCS 3-8 (median) & 8 & 6 & \\
GCS 9-13 (median) & 13 & 9 & \\
GCS 14 and 15 (median) & 15 & 15 & 0.06 \\
I\&V at referral & $10(25.6 \%)$ & $15(46.9 \%)$ & 0.08 \\
ICU admission & $20(51.3 \%)$ & $23(69.7 \%)$ & 0.62 \\
GCS mean (ICU) & 8.1 & 7.5 & 0.31 \\
GCS median (ICU) & 7 & 7 & \\
LOS (days) & $14.6(2-37)$ & $19.6(1-110)$ & $0.65)$ \\
Surgery & $28(71.8 \%)$ & $21(65.6 \%)$ & 0.58 \\
COVID-19 positive & N/A & 0 & \\
\hline
\end{tabular}

GCS Glasgow Coma Scale, ICU intensive care unit, $I \& V$ intubated and ventilated, $L O S$ length of stay, $R T C$ road traffic collision 
Table 2 Trauma diagnoses and aetiology

\begin{tabular}{lll}
\hline Diagnosis & $\mathbf{2 0 1 9}$ & $\mathbf{2 0 2 0}$ \\
\hline TBI & 40 & 36 \\
T-SAH & 4 & 1 \\
ASDH & 11 & 12 \\
CSDH & 2 & 2 \\
EDH & 5 & 10 \\
Contusion & 16 & 11 \\
IVH & 2 & 0 \\
Cranial fracture & 0 & 4 \\
Spinal trauma & 6 & 0 \\
Aetiology & & \\
Fall & $24(61.5 \%)$ & $10(31.3 \%)$ \\
High fall & $8(20.5 \%)$ & $12(37.5 \%)$ \\
RTC & $5(12.8 \%)$ & $4(12.5 \%)$ \\
Bicycle injury & 0 & $1(3.1 \%)$ \\
Alleged assault & $2(5.2 \%)$ & $5(15.6 \%)$ \\
\hline
\end{tabular}

$A S D H$ acute subdural hematoma, $C S D H$ chronic subdural hematoma, $E D H$ extradural hematoma, IVH intraventricular haemorrhage, $R T C$ road traffic collision, TBI traumatic brain injury, $T-S A H$ traumatic subarachnoid haemorrhage

and for severe TBI; the median GCS was 8 in 2019 and 6 in 2020 (Table 1).

\section{ICU admission, intubation, and ventilation status and GCS of ICU patients}

In 2019, 20 patients (51.3\%) were admitted to ICU. This increased to 23 patients $(69.7 \%)$ in $2020(p=0.08)$. Exactly $10(25.6 \%)$ and $15(46.9 \%)$ of patients were intubated and ventilated in 2019 and 2020 respectively $(p=0.06)$.

The mean initial GCS in ICU patients was 8.1 and 7.5 in 2019 and $2020(p=0.62)$. Median initial GCS of ICU patients was 7 in both years (Table 1).

\section{COVID-19 status}

No patients tested positive for COVID-19 in the time period (Table 1).

\section{Length of stay}

The mean LOS in 2019 was 14.6 days, and this increased to 19.6 days in $2020(p=0.31)$. The longest LOS was 110 days in 2020, and this increased the mean LOS considerably for that year (Table 1).

\section{Thirty-day mortality}

The 30-day mortality rate in 2019 and 2020 was 7.7 and $15.6 \%$ respectively $(p=0.45)$ (Table 3$)$.

\section{Patients undergoing surgery}

In 2019, 28 patients (71.8\%) had surgery. This was slightly reduced in 2020, with $21(65.6 \%)$ of patients requiring surgical intervention $(p=0.58)$ (Table 1).

\section{Mean time from acceptance to admission and from admission to surgery}

There was no statistically significant difference in the mean time from acceptance to admission. This was $4 \mathrm{~h} 58 \mathrm{~min}$ in 2019 and $3 \mathrm{~h} 55 \mathrm{~min}$ in 2020 respectively $(p=0.22)$.

The mean time from admission to surgery as $9 \mathrm{~h} 10 \mathrm{~min}$ in 2019 and $5 \mathrm{~h} 37 \mathrm{~min}$ in 2020 respectively $(p=0.35)$ (Table 3). Two patients were left out of the analysis for time from admission to theatre in 2020. They were transferred for neuro-observation. Unfortunately, they clinically deteriorated and required surgical intervention at 7 and 9 days post admission respectively.

\section{Discussion}

Our study reports a trend toward increased 30-day mortality that does not reach statistical significance (7.7 vs $15.6 \%$, $p=0.45$ ) when comparing trauma patients admitted during the first COVID-19 period to a year previous. Of the patients admitted due to trauma, $71.8 \%$ underwent an operation in 2019 and $65.6 \%$ in 2020 . There was a $17.9 \%$ decrease in
Table 3 Key performance indicator outcomes

\begin{tabular}{|c|c|c|c|}
\hline Outcomes & 2019 & 2020 & $p$ value \\
\hline Mortality (30 days) & $3(7.7 \%)$ & $5(15.6 \%)$ & 0.45 \\
\hline Transferred from other hospital & $35(89.7 \%)$ & $29(90.6 \%)$ & \\
\hline $\begin{array}{l}\text { Mean time from acceptance to admission } \\
\text { (hours:minutes) }\end{array}$ & $4: 58(1: 23-16: 54)$ & $3: 55(1: 06-10: 08)$ & 0.22 \\
\hline $\begin{array}{l}\text { Mean time from admission to surgery } \\
\text { (hours:minutes) }\end{array}$ & $9: 10(0: 35-47: 36)$ & $5: 37(0: 09-37: 56)^{*}$ & 0.35 \\
\hline
\end{tabular}

Two patient's time's were left out of the analysis for time from admission to theatre in 2020 as they clinically deteriorated after a period of neuroobservation and required surgical intervention at 7 and 9 days post admission, respectively 
trauma admissions during the 3-month COVID period of this year. COVID-19 has had a significant impact on both the volume and mechanism of trauma admissions to the National Neurosurgical Centre in Ireland. Trauma admissions to ICU remained steady despite an overall reduction in trauma referrals and admissions. Transfer time, time to surgery, and length of stay were impacted by COVID-19.

COVID-19 has posed unique challenges in the surgical treatment of patients across all specialities. A report by the GlobalSurg group found that in patients undergoing elective or emergency surgery with a pre-operative diagnosis of COVID-19, mortality rates were increased at 23.8\% [15]. In our trauma cohort comparing a 3-month COVID-19 period and the same period 1 year before, there was a trend toward increased 30-day mortality (7.7 vs $15.6 \%$ ) in the COVID period that was not statistically significant. Both these mortality rates are less than an Irish study that reported a mortality rate of $25.5 \%$ in patients undergoing emergency neurosurgical intervention for TBI that were admitted to ICU between July 2012 and December 2015 [3]. This may reflect improvement in trauma care in the intervening period, differences in TBI severity, or institutional differences in TBI management. COVID has had varying impacts on neurosurgical and neurotrauma patients worldwide. Emergent and non-emergent neurosurgical referrals and admissions have declined [10, 11, 16, 17]. In contrast, TBI admission numbers to ICU have remained steady [11, 18]. Mortality rates of 1.1 and $2.0 \%$ were reported in all referrals and admissions to a tertiary neurosurgical unit in London in a non-COVID and COVID period [19]. Similarly, in patients admitted to a neurosurgical ICU in Finland with TBI or aneurysmal subarachnoid haemorrhage (SAH), no difference in mortality was seen (in either diagnosis) [18]. In contrast, a study in India reported a significant increase in neurotrauma mortality during the pandemic and attributes this to more severe injury mechanism and polytrauma [20]. An alarming trend that has become apparent is the delay in presentation and resulting worse outcomes for time sensitive emergencies such as spinal emergencies [11]. This is not unique to neurosurgery and has been an issue in the treatment ischaemic stroke [21]. Beyond neurosurgery, the mortality results are also mixed. A study from New Zealand reported an overall decrease in emergency general surgery admissions, but observed no difference in mortality from a COVID and comparison time period [12]. In contrast, Surek et al. [22] report a significant increase from 1.2 to $4.9 \%$ mortality in emergency general surgery during the COVID period.

An important and increasingly recognised consequence of COVID-19 lockdowns has been a delay in presentation to medical attention. In the present study, there was a trend toward increased mortality during lockdown that was not statistically significant. Hecht et al. [11] found there were delays in presentation of spinal emergencies and chronic subdural hematomas (cSDH). Of concern is the fact that symptoms were more severe and outcomes were more likely to be worse in cSDH cases in the COVID period. Presentation delays have been reported in subarachnoid haemorrhage, cervical epidural abscess with quadriplegia, and paediatric tumour causing oedema and coma [23]. The observed increase in mortality in our study may partly be due to a delay in presentation of patients due to fear of contracting COVID-19, and other studies with increased mortality suggest this may be a contributor [20,22]. Initial GCS is an independent predictor of neurological outcome and mortality. The lower the initial GCS, the more likely there will be a poor outcome [24]. The mean initial GCS at referral was lower (9.1) in 2020 compared to 10.6 in 2019. The median GCS was also lower at 9 in 2020 versus 13 in 2019. The percentage of patients intubated and ventilated in 2020 was $46.9 \%$. This figure was only $25.6 \%$ in 2019 . The proportion of patients requiring ICU admission was also increased from 51.3 to $69.7 \%$ during the COVID time period. These imply a more severe injury in 2020, yet the proportion of patients with a GCS of 3-8 was actually higher in 2019. However, the median GCS of moderate ( 9 vs 13 ) and severe (6 vs 8 ) TBI are lower in 2020 than 2019. The average LOS was 5 days longer in 2020 compared to 2019 (19.6 vs 14.6 days). In other words, the moderate TBI cohort of patients in 2020 had a more severe injury compared the cohort in 2019 , and therefore were more likely to have a poor outcome. This promotes the hypothesis that the severity of trauma at presentation was higher in 2020 than 2019 and may explain the increased mortality rate in 2020 . Other factors that may contribute to the increased mortality include a delay in presentation and a change in the mechanism of injury. In admitted patients, there were proportional increases in high falls and alleged assaults and a decrease in falls in 2020. These are higher energy mechanisms of injury, increasing the risk of a more severe injury.

The transfer time and time from admission to surgery were not adversely affected by COVID-19. In fact, on average, these times improved in 2020. The fastest transfer and time to surgery times were $1 \mathrm{~h}$ and $6 \mathrm{~min}$ and $9 \mathrm{~min}$ respectively, both occurring in 2020. The COVID-19 status of patients could potentially lead to delays to theatre. No patients tested positive on for COVID-19 in the cohort. This is somewhat surprising. Routine COVID-19 testing for patients undergoing surgery was not in place for all of the time period studied. More relevant is the fact that these are time sensitive emergency surgeries and it would not be appropriate to wait for the result of a COVID swab. Therefore, in patients going to theatre with an unknown COVID19 status, the following precautions were employed; for theatre staff, N95 masks, goggles, and surgical hoods were used. During intubation and extubation and 10 min after these interventions, only anaesthetics staff were present in 
theatre. In the provision of neuro-oncological operations in our institution, these results were replicated and no delays in transfer time or time to surgery were found [25].

We observed a $17.9 \%$ decrease in neurotrauma patients admitted to a National Neurosurgical Centre during a 3-month COVID-19 lockdown period. Similar to mortality, there were variations in neurosurgical trauma and emergency admissions numbers during COVID-19 time periods depending on cohort, with the majority observing an overall decrease [11, 18, 20, 26-28]. In our study, the number admitted to ICU, overall number, and proportion of patients admitted actually increased in TBI and trauma patients in the COVID period. It is not significantly increased but the relatively small sample size may explain this result. This has important resource implications. Ireland has 5.2 publicly funded ICU beds per 100,000 population, less than half of the 11.5 beds per 100,000 European average [29]. As numbers of COVID19 cases in a country increase, the numbers of hospital and ICU admissions similarly increase. ICU beds are a scarce resource providing specialist care to patients, with severe COVID-19 infections requiring ICU admission and ventilator support. This underfunding of critical care beds and lack of resources may have contributed to the increased mortality rate in our study. Severe COVID-19 infections place a high burden on a limited resource. Combatting a novel virus may have required an increased focus on COVID over neurosurgical patients. This was likely exacerbated by reduction in staff numbers. ICU and neurosurgical workforces were depleted at various stages by contracting the virus and being close contacts leading to mandatory self-isolation. As we have shown, at least $50 \%$ of the TBI and trauma cases admitted require ICU care. In addition, other critically ill neurosurgical patients, e.g. aneurysmal SAH and hydrocephalus, require ICU admission. These pathologies will continue to require a significant number of ICU bed days during or after any lockdown restrictions.

This study has several limitations. It is a retrospective observational study. While it is a single centre study, the hospital is the national neurosurgical centre. We have not investigated presentation delays in TBI and spinal injury cases. This will be of interest in future studies. We have reported mortality data but not the functional outcomes of trauma patients that were admitted. In future investigations, patient functional outcomes could be included and patients followed up in the short to medium term to assess the physical and psychological effects of their TBI or spinal injuries.

\section{Conclusions}

Traumatic brain injury and spinal injury mortality rates in patients admitted to the National Neurosurgical Centre were increased during the first COVID-19 lockdown period in
2020, although the numbers did not reach statistical significance. There was a $17.9 \%$ decrease in trauma admissions observed. Trauma admission rates to ICU remained unchanged despite an overall reduction in trauma referrals and admissions. Transfer time, time to surgery, and length of stay were impacted by COVID-19. Despite the challenges COVID-19 has posed, it is important to maintain a fully functioning neurosurgical and neurocritical care service during the pandemic.

\section{Declarations}

Conflict of interest The authors declare no competing interests.

\section{References}

1. Finfer SR, Cohen J (2001) Severe traumatic brain injury. Resuscitation 48(1):77-90

2. Majdan M, Plancikova D, Brazinova A et al (2016) Epidemiology of traumatic brain injuries in Europe: a cross-sectional analysis. Lancet Public Health 1(2):e76-e83

3. Amoo M, O'Halloran PJ, Leo AM et al (2018) Outcomes of emergency neurosurgical intervention in neuro-critical care patients with traumatic brain injury at Cork University Hospital. Br J Neurosurg 32(6):585-589

4. Ryu JA, Yang JH, Chung CR et al (2017) Impact of neurointensivist co-management on the clinical outcomes of patients admitted to a neurosurgical intensive care unit. J Korean Med Sci 32(6): 1024-1030

5. Andriessen TM, Horn J, Franschman G et al (2011) Epidemiology, severity classification, and outcome of moderate and severe traumatic brain injury: a prospective multicenter study. J Neurotrauma 28(10):2019-2031

6. Mulcahy LT, O'Halloran PJ, O'Rourke C et al (2020) Traumatic brain injury in the Republic of Ireland twenty-five years on: a comparison of two cohorts from a neurosurgical unit. Brain Inj 34(12):1610-1617

7. Department of Health (2020) Latest updates on COVID-19 (Coronavirus) https://www.gov.ie/en/news/7e0924-latest-updates-oncovid-19-coronavirus/\#the-latest-news-as-of-545pm-on-monday22-june Accessed 28 April 2021

8. Department of the Taoiseach (2020) Statement by An Taoiseach on measures to tackle Covid-19 https://www.gov.ie/en/speech/ 5a280b-statement-by-an-taoiseach-on-measures-to-tackle-covid19-washington/ Accessed 15 Septmeber 2020

9. Department of the Taoiseach (2020) Easing the COVID-19 restrictions on 18 May (Phase 1) https://www.gov.ie/en/publication/ ad5dd0-easing-the-covid-19-restrictions-on-may-18-phase-1/ Accessed 27 October 2020

10. Horan J, Duddy JC, Gilmartin B et al (2021) The impact of COVID-19 on trauma referrals to a national neurosurgical centre. Ir J Med Sci 7:1-13

11. Hecht N, Wessels L, Werft FO et al (2020) Need for ensuring care for neuro-emergencies-lessons learned from the COVID-19 pandemic. Acta Neurochir (Wien) 162(8):1795-1801

12. McGuinness MJ, Hsee L (2020) Impact of the COVID-19 national lockdown on emergency general surgery: Auckland City Hospital's experience. ANZ J Surg 90(11):2254-2258

13. Christey G, Amey J, Campbell A et al (2020) Variation in volumes and characteristics of trauma patients admitted to a level one 
trauma centre during national level 4 lockdown for COVID-19 in New Zealand. N Z Med J 133(1513):81-88

14. Census. Central Statistics Office (2019) StatBank census populations. Central Statistics Office. https://statbank.cso. $\mathrm{ie} / \mathrm{px} / \mathrm{pxeirestat} /$ Statire/SelectVarVal/Define.asp?Maintable= EY029\&Planguage $=0$. A

15. COVIDSurg Collaborative (2020) Mortality and pulmonary complications in patients undergoing surgery with perioperative SARS-CoV-2 infection: an international cohort study Lancet 396(10243):27-38

16. Ahuja S, Shah P, Mohammed R (2021) Impact of COVID-19 pandemic on acute spine surgery referrals to UK tertiary spinal unit: any lessons to be learnt? Br J Neurosurg 35(2):181-185

17. Figueroa JM, Boddu J, Kader M et al (2021) The effects of lockdown during the severe acute respiratory syndrome coronavirus 2 (SARS-CoV-2) pandemic on neurotrauma-related hospital admissions. World Neurosurg 146:e1-e5

18. Luostarinen T, Virta J, Satopää J et al (2020) Intensive care of traumatic brain injury and aneurysmal subarachnoid hemorrhage in Helsinki during the Covid-19 pandemic. Acta Neurochir (Wien) 162(11):2715-2724

19. Ashkan K, Jung J, Velicu AM et al (2020) Neurosurgery and coronavirus: impact and challenges-lessons learnt from the first wave of a global pandemic. Acta Neurochir (Wien) 1-13

20. Goyal N, Swain SK, Gupta K et al (2020) "Locked up inside home" - head injury patterns during coronavirus disease of 2019 pandemic. Surg Neurol Int 11:395

21. Montaner J, Barragán-Prieto A, Pérez-Sánchez S et al (2020) Break in the stroke chain of survival due to COVID-19. Stroke 51(8):2307-2314

22. Surek A, Ferahman S, Gemici E et al (2020) Effects of COVID-19 pandemic on general surgical emergencies: are some emergencies really urgent? Level 1 trauma center experience. Eur J Trauma Emerg Surg 1-6

23. Tartara F, Cofano F, Zenga F et al (2020) Are we forgetting nonCOVID-19-related diseases during lockdown? Acta Neurochir (Wien) 162(7):1501

24. Narayan RK, Greenberg RP, Miller JD et al (1981) Improved confidence of outcome prediction in severe head injury. A comparative analysis of the clinical examination, multimodality evoked potentials, CT scanning, and intracranial pressure. J Neurosurg 54(6):751-62

25. Amoo M, Horan J, Gilmartin B et al (2020) The provision of neuro-oncology and glioma neurosurgery during the SARS-CoV-2 pandemic: a single national tertiary centre experience. Ir J Med Sci 1-7

26. Antony J, James WT, Neriamparambil AJ et al (2020) An Australian response to the COVID-19 pandemic and its implications on the practice of neurosurgery. World Neurosurg 139:e864-e871

27. Lubansu A, Assamadi M, Barrit S et al (2020) COVID-19 impact on neurosurgical practice: lockdown attitude and experience of a European academic center. World Neurosurg 144:e380-e388

28. Mathiesen T, Arraez M, Asser T et al (2020) A snapshot of European neurosurgery December 2019 vs. et al March 2020 just before and during the Covid-19 pandemic Acta Neurochir (Wien) 162(9):2221-2233

29. National Office of Clincal Audit (2020) Major trauma audit national report $2018 \mathrm{https} / / / \mathrm{www}$.noca.ie/documents/majortrauma-audit-national-report-2018 Accessed 21 June 2020

Publisher's Note Springer Nature remains neutral with regard to jurisdictional claims in published maps and institutional affiliations. 